\title{
Détermination de la tension des câbles à partir des fréquences de vibration
}

\author{
Dominique Siegert* — Marta Magdalena Choinska** \\ Pierre Brevet $^{* * *}$ — Daniel Bruhat ${ }^{* * *}$ — François Toutlemonde*
}

Laboratoire Central des Ponts et Chaussées

* FDOA - 58, boulevard Lefebvre, F-75732 Paris cedex 15

** R\&DO, Institut de Recherche GeM

Ecole Centrale de Nantes -Université de Nantes - CNRS UMR 6183

1 rue de la Noё, BP 92101, F-44321 Nantes cedex 3

Marta.Choinska@ec-nantes.fr

*** MACOA - Route de Bouaye, BP 19, F-44340 Bouguenais

Dominique.Siegert@lcpc.fr

RÉSUMÉ. La détermination de la tension dans les câbles et armatures de génie civil à partir des fréquences de vibration mesurées est considérée comme simple à mettre en ouvre lorsque la relation entre la tension et les fréquences de vibration est donnée par le modèle de la corde vibrante. La contribution proposée dans cet article concerne l'étude de modèles mécaniques simples pour évaluer les effets des conditions d'ancrage représentées par des liaisons élastiques et des distributions d'inertie. L'application des techniques de recalage de modèle à partir des premières fréquences propres mesurées permet d'étendre le domaine d'application de la méthode basée sur le modèle de la corde vibrante à des cas où il ne convient plus. Le recalage des modèles étudiés repose sur l'identification des conditions d'encastrement élastiques, de la rigidité à la flexion des câbles et du fonctionnement des dispositifs d'ancrage articulés. Les exemples d'application présentés sont issus de mesures sur des dispositifs de précontrainte, de suspension et de haubanage d'ouvrages en service.

ABSTRACT. When the relationship between the tension force and the eigen frequencies is given by the model of the vibrating cord, the determination of the tension in stay and external prestressing cables from measured vibration frequencies may be relatively easy. This paper deals with simple mechanical models of anchorage conditions. The model updating method based on the sensitivity of the vibration frequencies is used to identify the boundary conditions and the bending rigidity of the cable. Thus, the method based on the vibrating cord model has been extended to cases where it was no longer applicable. Case studies of short stay-cables and external pre-stressing bars are presented.

MOTS-CLÉS : hauban, précontrainte extérieure, tension, vibration, fréquence, analyse modale, recalage de modèle, identification de paramètres.

KEYWORDS: stay-cable, external prestressing, tension-force, vibration, frequency, modal analysis, model updating, parameter identification. 


\section{Introduction}

La méthode de détermination des valeurs de tension dans les câbles de génie civil (haubans et précontrainte extérieure) à partir des fréquences mesurées des vibrations libres est couramment utilisée. Cette méthode, basée sur l'application du modèle de la corde vibrante, a la réputation méritée d'être actuellement la plus simple à mettre en œuvre pour le contrôle des tensions dans les câbles de haubanage et de précontrainte extérieure (Ph. De Mars, D. Hardy (1985); J.L. Robert, D. Bruhat, J.P. Gervais (1991)).

Les travaux pour justifier le domaine d'utilisation de cette technique ont abouti à la rédaction de la méthode d'essai LPC n³5 (J.L. Robert (1993)). Le principe de la méthode consiste à mettre le câble en vibration par une excitation transitoire et à mesurer la réponse libre à l'aide d'un accéléromètre. Les fréquences propres sont obtenues par le calcul numérique de la transformée de Fourier du signal d'accélération. L'expression des fréquences propres dans le modèle de la corde vibrante s'écrit :

$$
f_{n}=\frac{n}{2 L} \sqrt{\frac{T}{m}}
$$

où $\mathrm{n}(=1,2,3, \ldots)$ représente l'ordre du mode de vibration, $\mathrm{L}$ - la longueur entre extrémités fixes, $\mathrm{T}$ - la tension dans le câble et $\mathrm{m}$ - la masse linéique du câble. Dans la suite on notera $f$, la fréquence fondamentale de vibration calculée avec le modèle de la corde vibrante (correspondant à $\mathrm{n}=1$ ).

Pour appliquer le modèle de la corde vibrante avec une erreur de modèle sur la tension inférieure à $2 \%$, la méthode d'essai recommande de vérifier que l'écart entre les 7 premières fréquences propres mesurées est constant. La rigidité à la flexion du câble étant une cause de dispersion, on doit également vérifier par le calcul que l'effet de la rigidité à la flexion peut être négligé. Le calcul est basé sur le modèle de la poutre tendue parfaitement encastrée, la condition pour utiliser le modèle de la corde vibrante avec la première fréquence de vibration s'écrit alors :

$$
\varepsilon=\frac{E I}{T \cdot L^{2}} \leq 2,5 \cdot 10^{-5}
$$

Pour les câbles peu tendus ou de longueur importante, il faut prendre en compte l'effet du poids propre pour calculer la première fréquence de vibration dans le plan vertical (SETRA (2001)). La correction sur la première fréquence propre s'exprime en fonction du rapport de la flèche due au poids propre à la portée $(\mathrm{s} / \mathrm{L})$ et du produit EA du module axial E [MPa] et de la section A $\left[\mathrm{mm}^{2}\right]$ du câble :

$$
f=f_{c v} \sqrt{1+\frac{512}{\pi^{4}}(s / L)^{2}(E A / T)} \quad \text { avec } \quad \frac{s}{L}=\frac{m g L \cos (\beta)}{8 T}
$$


où $\beta$ est l'inclinaison du hauban par rapport à l'horizontale et $g$ vaut $9,81\left[\mathrm{~m} / \mathrm{s}^{2}\right]$. Notre contribution vise à examiner avec des modèles mécaniques simples l'effet des conditions d'ancrage pour préciser et étendre le domaine d'application de la méthode des vibrations à la détermination de tensions. Il s'agit d'abord de proposer des compléments de vérification pour apprécier l'erreur de modélisation susceptible d'être commise lorsque la relation de non dispersion n'est pas ou ne peut pas être vérifiée jusqu'au septième mode. Dans cette démarche, on cherche alors à vérifier que l'utilisation de la relation entre la tension et la première fréquence propre du modèle de la corde vibrante est justifiée. Comme cette approche est limitée, il s'agit ensuite d'étendre le principe de la méthode aux éléments tendus trop courts pour que le modèle de la corde vibrante puisse convenir, il est alors nécessaire de mettre en œuvre des méthodes de recalage de modèles qui consistent à corriger les valeurs des paramètres choisis du modèle à partir d'une comparaison entre les premières fréquences mesurées et calculées.

Le modèle de la poutre tendue avec des encastrements élastiques est d'abord présenté. Ce modèle simple et classique permet déjà d'étendre le domaine d'application de la méthode vibratoire à la mesure de tension en prenant en compte les effets de la rigidité à la flexion de l'armature et les conditions d'encastrement schématisées par des liaisons élastiques. Ensuite l'effet d'inertie des dispositifs d'ancrage est étudié à partir d'un modèle simple du système constitué d'un culot d'ancrage monté sur chape. Ce dispositif d'ancrage représenté sur la figure 1 est assez souvent utilisé pour les câbles du type monotoron multicouche. La figure 1 montre l'utilisation de techniques plus ou moins sophistiquées pour réaliser la liaison d'articulation: selon la qualité d'articulation recherchée, l'axe peut être monté sur une rotule.

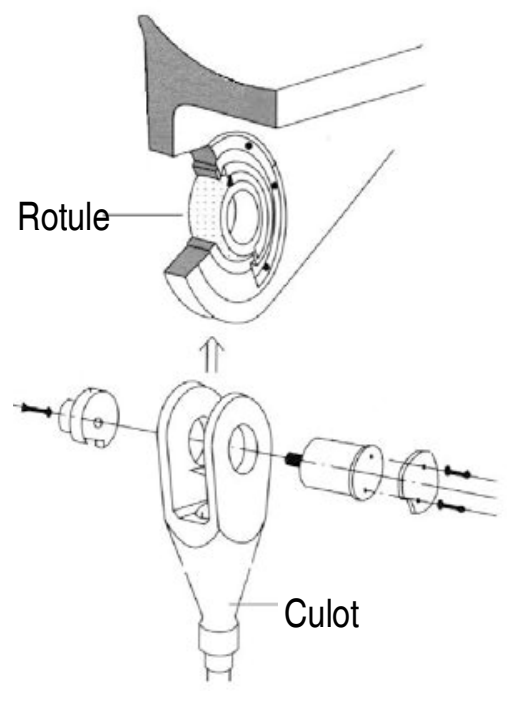

Figure 1. Dispositif d'ancrage (Akashi-Kaikyo bridge, 1998)

La valeur du module de rigidité à la flexion d'un câble n'est pas bien connue. Ce paramètre dépend de la géométrie de l'assemblage des fils et des conditions de contact entre les fils. La valeur maximale de la rigidité à la flexion est calculée en 
supposant que les fils ne glissent pas avec l'hypothèse de conservation des sections droites.

Des exemples d'applications à des cas expérimentaux issus de mesures réalisées sur des ouvrages réels sont présentés. Le premier exemple concerne une armature de précontrainte extérieure courte. Ensuite deux cas de câbles de haubanage montés sur culots d'ancrage à chape sont traités respectivement pour une longueur d'environ $10 \mathrm{~m}$ et $30 \mathrm{~m}$. La technique de recalage de modèles par éléments finis basée sur la méthode de sensibilité est utilisée pour déterminer les valeurs des paramètres inconnus du modèle que sont la tension, les constantes des liaisons élastiques et le module de rigidité à la flexion à partir des premières fréquences propres mesurées.

\section{Modélisation}

\subsection{Poutre tendue avec encastrements élastiques}

Le modèle de la poutre tendue dont les extrémités sont encastrées par des liaisons élastiques est représenté sur la figure 2. Les paramètres du modèle sont : la rigidité à la flexion $\mathrm{EI}$, la masse linéique $\mathrm{m}$, la longueur de la poutre $\mathrm{L}$, la constante élastique d'encastrement $\mathrm{C}$, la tension $\mathrm{T}$. Les premières fréquences propres $f_{n}$ sont alors calculées et peuvent être déterminées expérimentalement. La constante d'encastrement correspond à la relation linéaire entre le moment de flexion et la rotation de la poutre aux extrémités. Pour simplifier, on a considéré ici le cas de conditions symétriques.

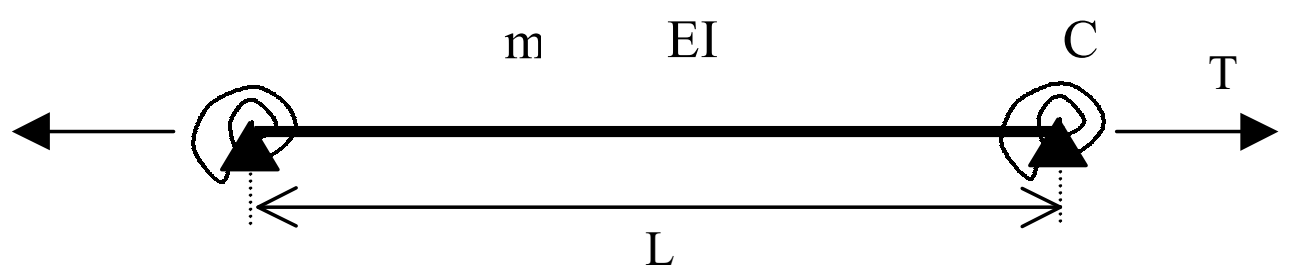

Figure 2. Poutre tendue avec extrémités élastiquement encastrées

Les dimensions des grandeurs du problème exprimées en fonction des grandeurs fondamentales notées $[\mathrm{M}],[\mathrm{L}],[\mathrm{T}]$ pour désigner respectivement la masse, la longueur et le temps sont rapportées dans le tableau 1. L'analyse dimensionnelle permet de préciser la forme des relations entre les premières fréquences propres des vibrations transversales et les paramètres du modèle. Ainsi, l'application directe du théorème de Buckingham conduit à écrire les relations recherchées sous la forme réduite avec $3(=6-3)$ paramètres sans dimension.

$$
\frac{f_{n}}{f_{c v}}=\Omega_{n}(\varepsilon, \alpha)
$$


avec $f_{c v}=\frac{1}{2 L} \sqrt{\frac{T}{m}} \quad \varepsilon=\frac{E I}{T L^{2}} \quad \alpha=\frac{C L}{E I}$

\begin{tabular}{|c|c|c|c|c|c|c|}
\hline & $\mathbf{f}$ & $\mathbf{E I}$ & $\mathbf{m}$ & $\mathbf{L}$ & $\mathbf{C}$ & $\mathbf{T}$ \\
\hline$[\mathbf{M}]$ & 0 & 1 & 1 & 0 & 1 & 1 \\
\hline$[\mathbf{L}]$ & 0 & 5 & -1 & 0 & 2 & 1 \\
\hline$[\mathbf{T}]$ & -1 & -2 & 0 & 1 & -2 & -2 \\
\hline
\end{tabular}

Tableau 1. Dimensions des paramètres du modèle

Lorsque le paramètre $\alpha$ est nul, la poutre est parfaitement articulée aux extrémités. La relation entre le paramètre $\varepsilon$ et les fréquences propres est alors donnée par:

$$
\frac{f_{n}}{f_{c v}}=n \sqrt{1+n^{2} \pi^{2} \varepsilon}
$$

Lorsque la valeur du paramètre $\alpha$ tend vers l'infini, la poutre est parfaitement encastrée. L'équation aux fréquences propres s'écrit :

$$
2\left(\frac{1}{\operatorname{ch}(z L)}-\cos (a L)\right)+\frac{\sin (a L) \operatorname{th}(z L)}{a L z L}\left(z L^{2}-a L^{2}\right)=0
$$

où $a L=\sqrt{\sqrt{\frac{1}{4 \varepsilon^{2}}+\frac{\pi^{2} \Omega_{n}^{2}}{\varepsilon}-\frac{1}{2 \varepsilon}}}$ et $z L=\sqrt{\sqrt{\frac{1}{4 \varepsilon^{2}}+\frac{\pi^{2} \Omega_{n}^{2}}{\varepsilon}+\frac{1}{2 \varepsilon}}}$ avec $\Omega_{n}=\frac{f_{n}}{f_{c v}}$

Les solutions de cette équation transcendante sont obtenues par résolution numérique. Morse et Ingard (1968) ont exprimé les solutions sous la forme approchée d'un développement asymptotique valable lorsque la valeur du paramètre adimensionnel $\varepsilon$ est très petit devant 1 . La correction sur le calcul de la première fréquence propre est alors donnée par la formule approchée :

$$
f_{1}=f_{c v}\left[1+2 \sqrt{\varepsilon}+\left(4+\frac{\pi^{2}}{2}\right) \varepsilon\right]
$$

Des compléments sur l'étude de l'approximation de Morse et Ingard concernant l'ordre du développement asymptotique et son application pour les modes de vibration plus élevés sont donnés dans l'ouvrage de Valette et Cuesta (1993). La solution asymptotique de Morse et Ingard montre que pour des câbles suffisamment longs et tendus, les premières fréquences propres sont peu sensibles aux détails des conditions d'encastrement et à la rigidité à la flexion. En suivant la méthode $\mathrm{LPCn}^{\circ} 35$, on détermine ainsi par exemple la longueur minimale pour appliquer le modèle de la corde vibrante à un toron de précontrainte de diamètre $15,7 \mathrm{~mm}$ tendu 
avec une tension égale à $30 \%$ de la force à la rupture, on trouve une valeur pratiquement égale à $14 \mathrm{~m}$.

La figure 3 montre les résultats des calculs des facteurs de correction de la première fréquence propre du modèle corde vibrante évalués en fonction du paramètre $\varepsilon$ pour des poutres tendues dont les extrémités sont encastrées et articulées aux deux extrémités. Il apparaît que la sensibilité à la rigidité à la flexion dépend fortement des conditions aux limites. Ainsi, on vérifie que dans la gamme des valeurs de $\varepsilon$ représentée $(<0,01)$, le modèle de la poutre parfaitement articulée est confondu avec celui de la corde vibrante, ce qui montre donc le peu de sensibilité à la valeur du module de rigidité à la flexion.

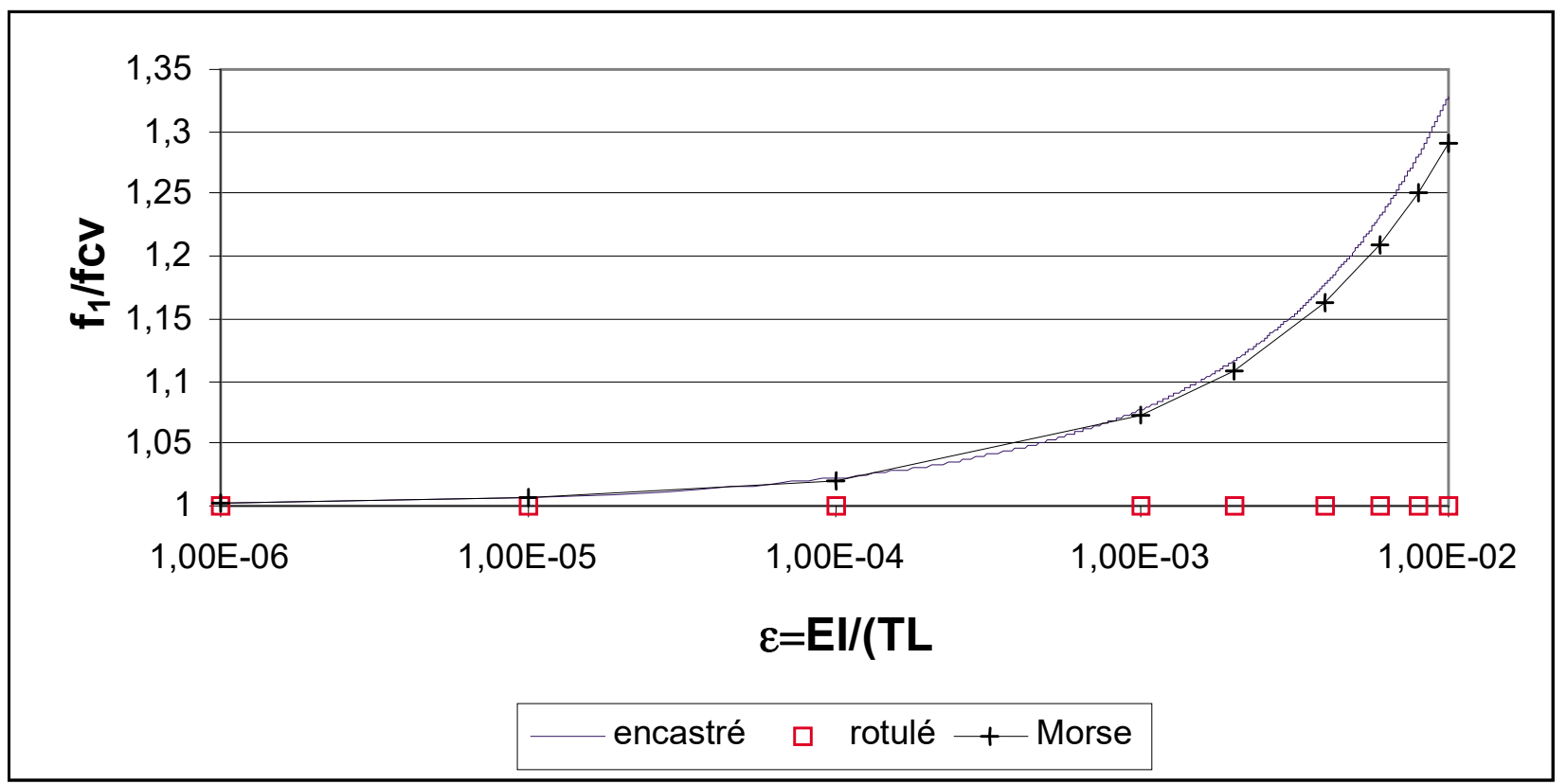

Figure 3. Calculs du facteur de correction sur la première fréquence propre

Sur la représentation graphique de la figure 3, on a également comparé la solution approchée de Morse et Ingard avec le résultat de la résolution numérique de l'équation aux fréquences propres analytique. Pour des encastrements parfaits, l'écart entre les résultats des deux calculs commence à être marqué pour une valeur du paramètre $\varepsilon$ supérieure à 0,001 .

La figure 4 montre le résultat des calculs par la méthode des éléments finis du facteur de correction de la fréquence propre fondamentale en fonction du paramètre d'encastrement élastique $\alpha$ pour une valeur du paramètre $\varepsilon$ égale à 0,005 .

L'influence des conditions aux limites et de la rigidité à la flexion dans la vibration des câbles est analogue aux phénomènes de couches limites en mécanique des fluides (Kevorkian J., Cole J.D.) : les effets de viscosité doivent être pris en compte dans les équations du mouvement au voisinage des conditions aux limites mais peuvent être négligés dans le domaine qui en est suffisamment éloigné. L'effet 
de la rigidité à la flexion des câbles est localisé dans la zone de flexion située au voisinage de l'extrémité encastrée.

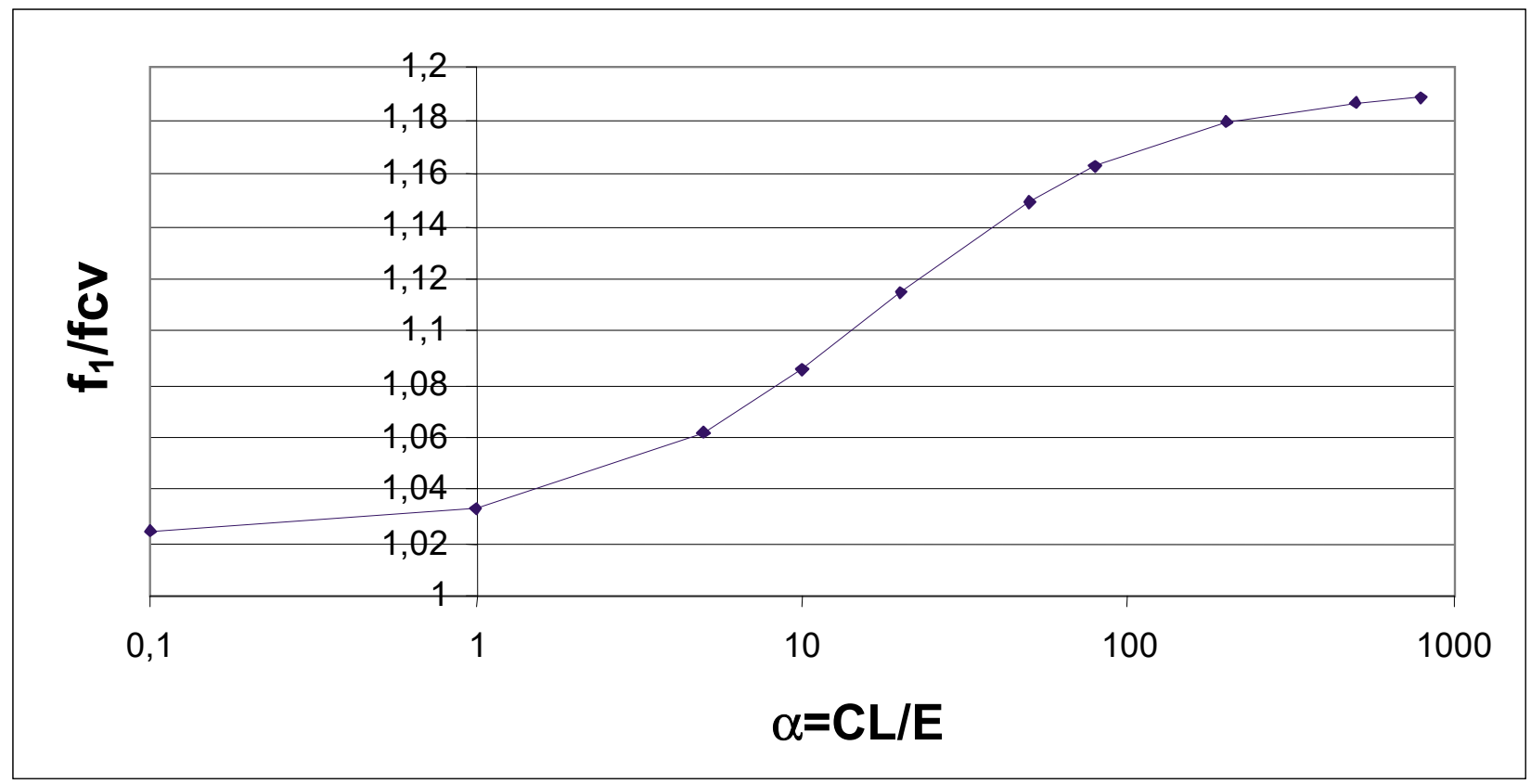

Figure 4. Effet de la de la constante d'encastrement élastique

\subsection{Effets d'inertie et de rigidité des systèmes d'ancrage}

Le modèle mécanique proposé d'un câble terminé à l'une de ses extrémité par un culot d'ancrage muni d'une chape est schématisé sur la figure 5.

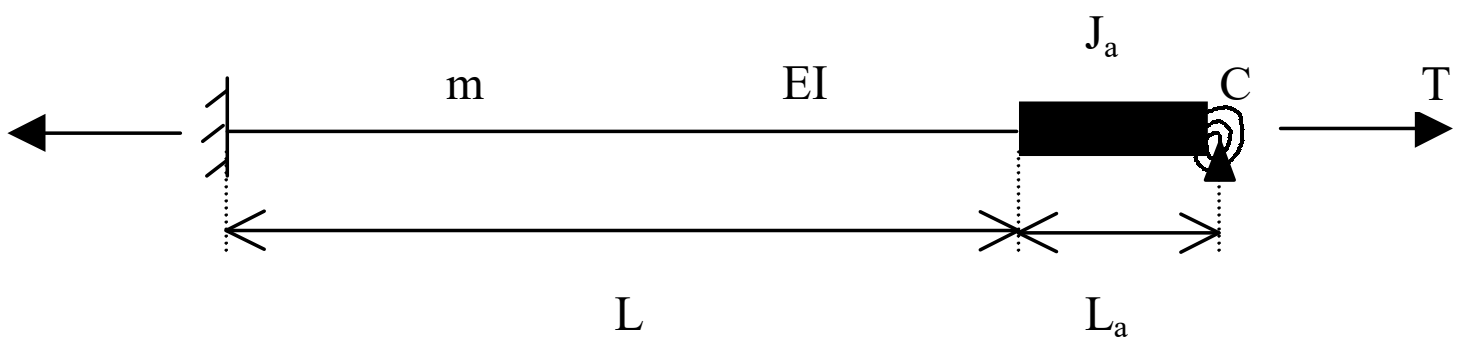

Figure 5. Schéma du modèle de la suspente

Le câble est supposé parfaitement encastré au niveau du culot d'ancrage et à l'autre extrémité fixe. Le dispositif d'ancrage est assimilé à un solide rigide caractérisé par un moment d'inertie en rotation $J_{a}$, une longueur $L_{a}$. Le système de chape monté sur un axe de rotation éventuellement par l'intermédiaire d'une rotule est schématisé par une articulation élastique de raideur C. La raideur est nulle pour une articulation parfaite sans frottement, l'autre cas limite correspond à une articulation parfaitement bloquée pour laquelle les déformations élastiques au niveau des contacts entre les éléments du système d'articulation sont négligés. Le 
fonctionnement partiellement bloqué de la rotule avec un moment de rappel élastique constitue une schématisation du comportement vibratoire du système pour des excitations suffisamment faibles pour ne pas activer les mécanismes de glissement ou de roulement au niveau de la liaison.

La méthode des éléments finis utilise des éléments de poutre tendue à deux nœuds (quatre degrés de liberté) pour réaliser des calculs de vibration dans un plan de flexion. Un nouvel élément avec trois degrés de liberté a été introduit pour représenter le couplage avec le culot d'ancrage dont la représentation schématique est montrée sur la figure 6 .

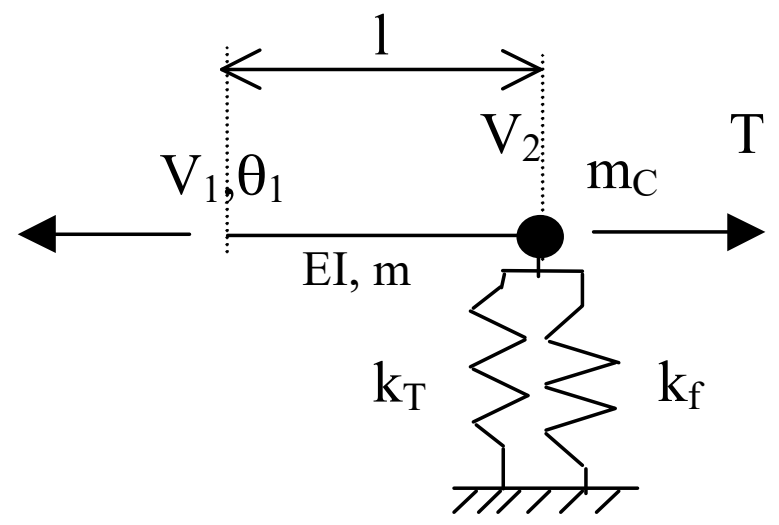

Figure 6. Elément couplé

$\left\{\mathrm{V}_{1}, \theta_{1}, \mathrm{~V}_{2}\right\}$ représente le vecteur constitué par le déplacement, la rotation du nœud 1 et le déplacement du nœud 2 montrés sur la figure 6 . La masse concentrée équivalente $\mathrm{m}_{\mathrm{c}}$ est associée à l'inertie du culot d'ancrage. La raideur concentrée équivalente correspond à un système de deux liaisons élastiques en parallèle $k_{T}$ et $k_{f}$ qui correspondent respectivement à la force de rappel géométrique produite par la tension et aux déformations locales au niveau des contacts dans la rotule liés aux frottements avant activation du glissement total. Les paramètres du modèle équivalent concentré s'expriment en fonction des paramètres représentés sur la figure 5 :

$$
m_{c}=\frac{J_{a}}{L_{a}^{2}} \quad k_{c}=k_{T}+k_{f} \quad \text { avec } \quad k_{T}=\frac{T}{L_{a}} \quad \text { et } \quad k_{f}=\frac{C}{L_{a}^{2}}
$$

Finalement, les matrices de rigidité et de masse de l'élément couplé s'écrivent :

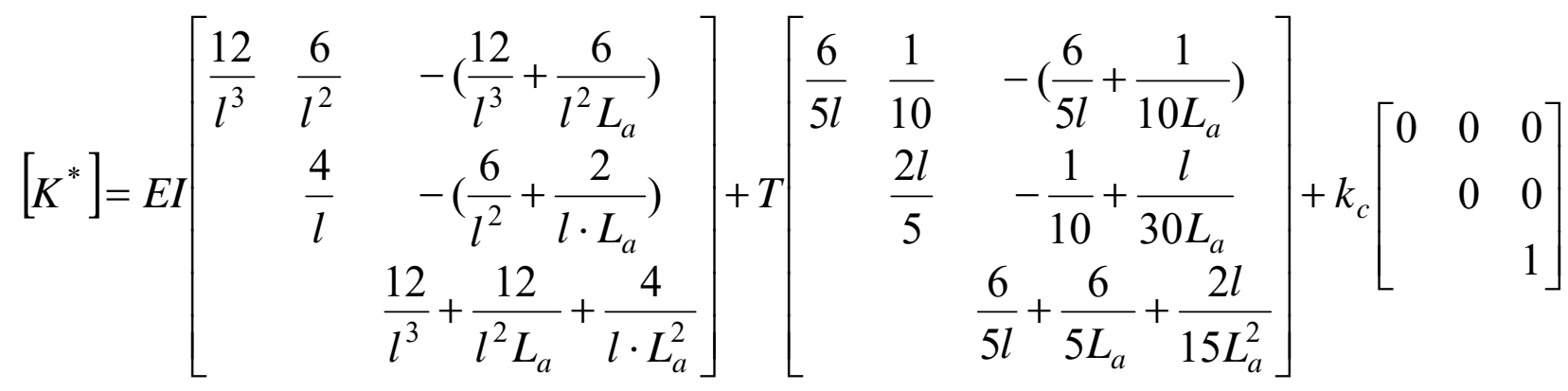




$$
\left[M^{*}\right]=m\left[\begin{array}{ccc}
\frac{13 l}{35} & \frac{11 l^{2}}{210} & \frac{9 l}{70}+\frac{13 l^{2}}{420 L_{a}} \\
& \frac{l^{3}}{105} & \frac{13 l^{2}}{420}+\frac{l^{3}}{140 L_{a}} \\
& \frac{13 l}{35}+\frac{22 l^{2}}{210 L_{a}}+\frac{l^{3}}{105 L_{a}}
\end{array}\right]+m_{c}\left[\begin{array}{ccc}
0 & 0 & 0 \\
& 0 & 0 \\
& & 1
\end{array}\right]
$$

Dans le cas d'une corde couplée parfaitement flexible $(E I=0)$, l'étude du modèle est simplifiée et on obtient l'expression analytique de l'équation aux fréquences propres :

$$
\tan (\pi x)=\frac{x}{\pi \cdot m^{*}\left(x^{2}-x^{* 2}\right)}
$$

où $x=\omega / \omega_{1}, m^{*}=\frac{M}{m \cdot L}, x^{*}=\frac{\sqrt{k_{c} / m_{c}}}{\omega_{1}}$ et avec $\omega_{1}=\frac{\pi \sqrt{T / m}}{L}$ la première pulsation propre de la corde vibrante dont les extrémités sont bloquées. Dans ce cas simplifié la résolution graphique de l'équation aux fréquences propres permet de visualiser les solutions, ce qui peut faciliter l'interprétation des résultats.

Quelques remarques supplémentaires peuvent être faites concernant l'utilisation du modèle de la corde tendue, couplée à l'une de ses extrémités :

a) Dans le cas de la chape parfaitement articulée, la tension ne figure plus dans l'équation aux fréquences propres qui s'exprime alors uniquement en fonction des caractéristiques géométriques et d'inertie du système.

b) L'effet de couplage se manifeste par un resserrement des fréquences propres inférieures à la fréquence $f_{c}$ du système masse ressort équivalent :

$$
f_{c}=\frac{1}{2 \pi} \sqrt{\frac{T \cdot L_{a}}{J_{a}}}
$$

Dans l'expression de cette fréquence, on reconnaît l'analogie avec la formule de la période de vibration d'un pendule pesant où $\mathrm{T}$ joue le rôle de la force de rappel à la place du poids.

Pour les fréquences propres supérieures, l'écart entre les fréquences successives augmente ensuite progressivement. L'effet du couplage sur les écarts entre les fréquences successives est d'autant plus marqué que la fréquence $f_{c}$ est proche des premières fréquences propres du système.

c) Si la longueur relative $l_{r}=L_{a} / L$ diminue suffisamment, les premières fréquences propres du système parfaitement articulé tendent vers les valeurs calculées avec le modèle de la corde vibrante dont la longueur libre inclut celle du dispositif d'ancrage. C'est d'ailleurs ce que recommande de faire la méthode 
$\mathrm{LPCn}^{\circ} 35$ pour tenir compte du dispositif d'ancrage en supposant le fonctionnement parfait de l'articulation. La première fréquence propre de vibration est alors approchée par :

$$
f_{1}=\frac{f_{c v}}{\left(1+l_{r}\right)}
$$

d) Le modèle simplifié de la corde couplée peut être utilisé pour évaluer de manière approchée l'effet sur la première fréquence propre d'autre types de dispositifs d'ancrage comme par exemple des culots d'ancrage montés sur des barres encastrées. Il suffit alors de déterminer le système masse - ressort équivalent pour le dispositif d'ancrage considéré avec une méthode de calcul approchée.

\subsection{Recalage des modèles par éléments finis}

La tension, les constantes de liaison élastique et éventuellement la distribution de rigidité à la flexion constituent les paramètres inconnus dans les modèles présentés. La méthode du recalage de modèle à partir des fréquences expérimentales permet de corriger une première estimation des valeurs des paramètres de façon à minimiser l'écart entre les fréquences calculées et les fréquences mesurées. Le modèle doit être suffisamment représentatif du comportement mécanique réel pour permettre un ajustement sur plusieurs des premiers modes. Les modes propres de vibration correspondent aux solutions du problème de valeurs propres qui s'écrit :

$$
\left(K^{-1} M-\lambda \cdot I\right)\{\Phi\}=0
$$

où $\{\Phi\}$ - vecteur propre des déplacements généralisés, $\lambda=\frac{1}{\omega^{2}}$ et $I$ - la matrice identité. Le problème d'optimisation consiste à minimiser la fonction objectif :

$$
J\left(p_{1}, p_{2}, \ldots, p_{p}\right)=\sum_{i=1}^{n}\left(\omega_{i}^{2}-\hat{\omega}_{i}^{2}\right)^{2}
$$

où $\mathrm{p}_{\mathrm{j}}$ sont les paramètres du modèle à identifier, $\omega_{i}$ sont les pulsations calculées, $\hat{\omega}_{i}$ sont les pulsations mesurées et $\mathrm{n}$ est le nombre de fréquences utilisées.

La condition nécessaire à vérifier s'écrit : $\left\{\frac{\partial J}{\partial p_{j}}\right\}=0$

La recherche de la solution consiste à itérer la résolution d'un problème linéaire des moindres carrés pour déterminer le vecteur correction (itération $\mathrm{k}+1$ ) sur les paramètres à identifier en fonction des fréquences propres calculées avec les valeurs des paramètres de l'itération $\mathrm{k}$ :

$$
S\{\delta p\}_{k+1}-\left\{\hat{\omega}_{i}^{2}-\left(\omega_{i}^{2}\right)_{k}\right\}=0
$$


où les composantes de la matrice de sensibilité sont obtenus par :

$$
S_{i j}=\frac{\partial \omega_{i}^{2}}{\partial p_{j}}=\left\{\Phi_{i}\right\}\left[\frac{\partial K}{\partial p_{j}}\right]\left\{\Phi_{i}\right\}
$$

avec $\left\{\Phi_{i}\right\}$ : vecteur propre des déplacements généralisés associé à la pulsation $\omega_{i}$ et normés par rapport à la masse.

Du point de vue pratique, il faut vérifier que le calcul converge vers l'optimum global après un nombre raisonnable d'itérations. La solution obtenue dépend d'une part du choix du modèle et des paramètres à identifier et d'autre part des incertitudes de mesure des fréquences. Nous avons présenté des modèles où seulement deux à trois paramètres (la tension, une constante de liaison élastique et la rigidité à la flexion du câble) doivent être évalués, il est donc nécessaire de mesurer au moins les 5 premières fréquences propres de vibration pour valider l'estimation en réduisant l'écart entre les fréquences propres calculées et mesurées. Dans le cas des câbles, la rigidité à la flexion considérée dans le problème de recalage correspond à la valeur définie au voisinage des ancrages compte tenu du peu de sensibilité des fréquences de vibration à la rigidité à la flexion en partie courante.

La méthode de recalage a été appliquée au cas de la poutre tendue partiellement encastrée par Livingston et al., (1995). Il en ressort que pour des essais réalisés en laboratoire l'erreur de recalage est inférieure à $5 \%$ lorsque la valeur du paramètre de flexion $\varepsilon$ est inférieure à 0,03 . Le recalage a été réalisé à partir des deux premières fréquences propres car il a été constaté une dégradation des résultats lorsque des modes plus élevés étaient pris en compte. L'erreur de recalage augmente également pour des valeurs du paramètre de flexion plus élevées. Néanmoins, cette valeur élevée du paramètre de flexion correspond seulement aux armatures les plus courtes que l'on peut rencontrer dans les ouvrages de génie civil.

\section{Exemples d'application}

\subsection{Barre de précontrainte extérieure transversale}

Des mesures des premières fréquences de vibration ont été réalisées sur une barre de précontrainte extérieure disposée transversalement dans un voussoir de pont en béton précontraint. Les caractéristiques de la barre en acier sont rapportées dans le tableau 2. La longueur de la barre est trop courte pour pouvoir envisager d'utiliser le modèle de la corde vibrante pour déterminer la tension. Le dispositif de fixation aux deux extrémités permet de penser que l'armature est encastrée de manière symétrique mais on ignore le degré d'encastrement. Le modèle avec deux paramètres à déterminer (la tension et la constante d'encastrement) de la poutre tendue avec extrémités élastiquement encastrées est donc utilisé. Les fréquences propres mesurées sont rapportées dans le tableau 3. 


\begin{tabular}{|c|c|c|c|c|}
\hline $\begin{array}{c}\text { Longueur } \\
\mathrm{L}[\mathrm{m}]\end{array}$ & $\begin{array}{c}\text { Masse linéique } \\
\mathrm{M}[\mathrm{kg} / \mathrm{m}]\end{array}$ & $\begin{array}{c}\text { Diamètre } \\
\mathrm{D}[\mathrm{mm}]\end{array}$ & $\begin{array}{c}\text { Module de } \\
\text { Young } \\
\mathrm{E}[\mathrm{MPa}]\end{array}$ & $\begin{array}{c}\text { Rigidité à la } \\
\text { flexion } \\
\left.\mathrm{EI} \mathrm{N} \cdot \mathrm{m}^{2}\right]\end{array}$ \\
\hline 6,35 & 6,31 & 32 & 210000 & 10809 \\
\hline
\end{tabular}

Tableau 2. Caractéristiques de la barre de précontrainte

\begin{tabular}{|c|c|c|c|c|c|c|}
\hline $\begin{array}{c}\text { Fréquences } \\
(\mathrm{Hz})\end{array}$ & $\mathrm{f}_{1}$ & $\mathrm{f}_{2}$ & $\mathrm{f}_{3}$ & $\mathrm{f}_{4}$ & $\mathrm{f}_{5}$ & $\mathrm{f}_{6}$ \\
\hline $\begin{array}{c}\text { Mesurées } \\
\text { (résolution) }\end{array}$ & 19,24 & 38,92 & 59,4 & 81,0 & 104,3 & 129,2 \\
\hline Calculées & $19,02)$ & $(0,04)$ & $(0,1)$ & $(0,1)$ & $(0,1)$ & $(0,1)$ \\
\hline
\end{tabular}

Tableau 3. Fréquences propres mesurées et calculées

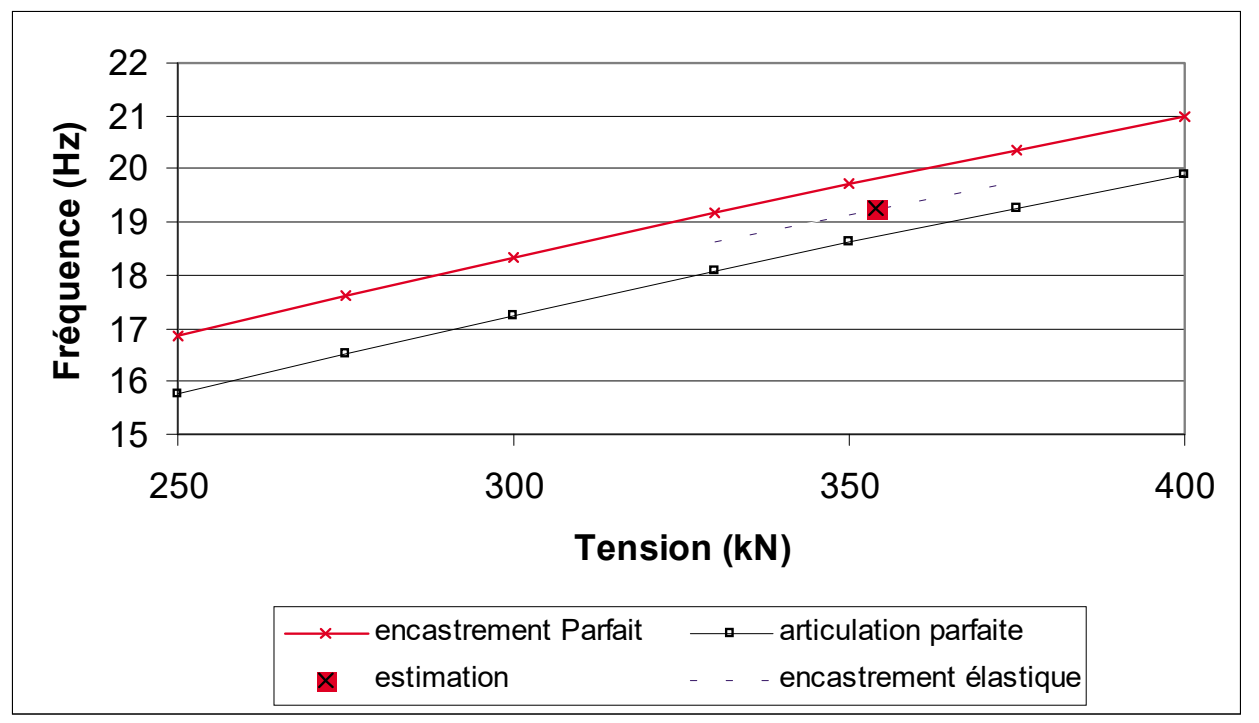

Figure 7. Relations entre la première fréquence de vibration et la tension pour les cas limites de conditions aux limites symétriques: encastrements parfaits, articulations parfaites

La tension déterminée après recalage du modèle à partir des trois premières fréquences propres mesurées est égale à $354 \mathrm{kN}$ et la valeur de la constante élastique d'encastrement est égale à 61,9 kN.m. L'écart relatif entre les tensions déterminées à partir des modèles avec extrémités parfaitement encastrées et parfaitement articulées est de $12 \%$. Les relations théoriques entre la première fréquence propre et la tension calculées pour des conditions aux limites d'encastrement parfait et d'articulation parfaite sont représentées sur la figure 7 . Le résultat du modèle recalé est également représenté sur la figure 7. Les valeurs des 6 premières fréquences de 
vibration calculées avec le modèle recalé sont rapportées dans le tableau 3. On constate que les écarts entre les fréquences mesurées et calculées sont inférieurs à la résolution expérimentale.

\subsection{Suspente courte d'un bow-string}

La figure 8 montre le culot d'ancrage à chape d'une suspente d'une longueur totale d'environ $12 \mathrm{~m}$ d'un pont de type Bow-String. Le dispositif montré correspond à l'ancrage en partie inférieure, l'ancrage réglable en partie supérieure est également constitué d'un culot, mais dont la face avant est en appui sur une surface plane fixe.

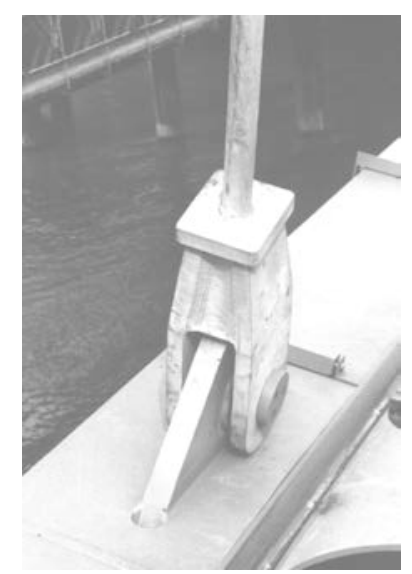

Figure 8. Dispositif d'ancrage à chape

Les caractéristiques de la suspente étudiée sont rapportées dans le tableau 4. Le module de rigidité à la flexion du toron a été calculé en supposant que les fils ne glissent pas ce qui donne une bonne estimation de la valeur en partie courante pour des amplitudes de vibration suffisamment faibles.

\begin{tabular}{|c|c|c|c|c|c|}
\hline \multicolumn{3}{|c|}{ Toron } & \multicolumn{3}{c|}{$\begin{array}{c}\text { Système d'ancrage } \\
\text { Culot rigide articulé }\end{array}$} \\
\hline $\begin{array}{c}\text { Diamètre } \\
{[\mathrm{mm}]}\end{array}$ & $\begin{array}{c}\text { Masse } \\
\text { linéique } \\
{[\mathrm{kg} / \mathrm{m}]}\end{array}$ & $\begin{array}{c}\text { Rigidité à la } \\
\text { flexion } \\
{\left[\mathrm{N} \cdot \mathrm{m}^{2}\right]}\end{array}$ & $\begin{array}{c}\text { Longueur } \\
\mathrm{L}[\mathrm{m}]\end{array}$ & $\begin{array}{c}\text { Inertie } \\
{\left[\mathrm{kg} \cdot \mathrm{m}^{2}\right]}\end{array}$ & $\begin{array}{c}\text { Longueur } \\
1_{\mathrm{d}}[\mathrm{m}]\end{array}$ \\
\hline 72 & 28,7 & 239000 & 10,83 & 26,875 & 0,605 \\
\hline
\end{tabular}

Tableau 4. Caractéristiques de la suspente

Les 6 premières fréquences propres mesurées pour des vibrations produites dans le plan perpendiculaire à l'axe de la chape sont rapportées dans le tableau 5. Avec la résolution de mesure indiquée sur le tableau 5, les mêmes valeurs de fréquences ont été obtenues pour une excitation dans le plan de l'axe de la chape. 


\begin{tabular}{|c|c|c|c|c|c|c|}
\hline Fréquences (Hz) & $\mathrm{f1}$ & $\mathrm{f} 2$ & $\mathrm{f3}$ & $\mathrm{f} 4$ & $\mathrm{f5}$ & $\mathrm{f6}$ \\
\hline $\begin{array}{c}\text { Mesurées } \\
\text { (résolution) }\end{array}$ & $\begin{array}{c}8,16 \\
(0,04)\end{array}$ & $\begin{array}{c}16,84 \\
(0,04)\end{array}$ & $\begin{array}{c}26,4 \\
(0,1)\end{array}$ & $\begin{array}{c}37,2 \\
(0,1)\end{array}$ & $\begin{array}{c}49,4 \\
(0,1)\end{array}$ & $\begin{array}{c}63,2 \\
(0,1)\end{array}$ \\
\hline $\begin{array}{c}\text { Calculées } \\
\text { Rotule articulée }\end{array}$ & 8,16 & 16,45 & 25,05 & 34,60 & 45,94 & 59,41 \\
\hline $\begin{array}{c}\text { Calculées } \\
\text { Rotule bloquée }\end{array}$ & 8,16 & 16,99 & 27,04 & 38,73 & 52,32 & 67,99 \\
\hline Recalage 2 paramètres & 8,16 & 16,84 & 26,40 & 36,83 & 47,73 & 59,41 \\
\hline Recalage 3 paramètres & 8,17 & 16,83 & 26,40 & 37,2 & 49,40 & 63,02 \\
\hline
\end{tabular}

Tableau 5. Fréquences de vibration mesurées et calculées

Le modèle retenu pour interpréter les résultats des mesures correspond au schéma de la figure 5. Les liaisons d'encastrement du toron dans le culot d'ancrage sont supposées parfaites et la rigidité à la flexion du toron égale à sa valeur maximale. Comme dans le cas du modèle de la poutre partiellement encastrée traitée dans le paragraphe qui précède, deux paramètres sont alors à identifier : la tension et la constante de rappel élastique définie au niveau de la rotule du dispositif d'ancrage bas.

Les tensions ont été respectivement calculées avec la première fréquence propre mesurée pour les cas limites correspondant à une articulation parfaite au niveau de la chape et une articulation complètement bloquée ce qui revient à considérer le toron encastré à ses deux extrémités. La tension obtenue avec une rotule parfaite est égale à $906 \mathrm{kN}$, elle est égale à $693 \mathrm{kN}$ lorsque la rotule est bloquée. L'écart relatif entre les deux déterminations est de $30 \%$.Les 6 premières fréquences propres calculées avec les deux hypothèses de fonctionnement de la rotule sont rapportées dans le tableau 5.

La résolution du problème de recalage de modèle par la méthode de sensibilité nous a permis de déterminer à partir des trois premières fréquences mesurées les valeurs de la tension et de la constante élastique de rappel respectivement égales à $747 \mathrm{kN}$ et $6907 \mathrm{kN} . \mathrm{m}$. Les valeurs des 6 premières fréquences calculées avec ce premier modèle recalé sont rapportées dans le tableau 5. On constate qu'à partir du quatrième mode de vibration l'écart entre les fréquences calculées et mesurées augmente. La figure 9 montre une représentation graphique des isovaleurs des trois premières fréquences dans le plan défini par la tension et la constante élastique de rappel. On vérifie graphiquement que l'intersection des trois courbes est définie en un point unique. 


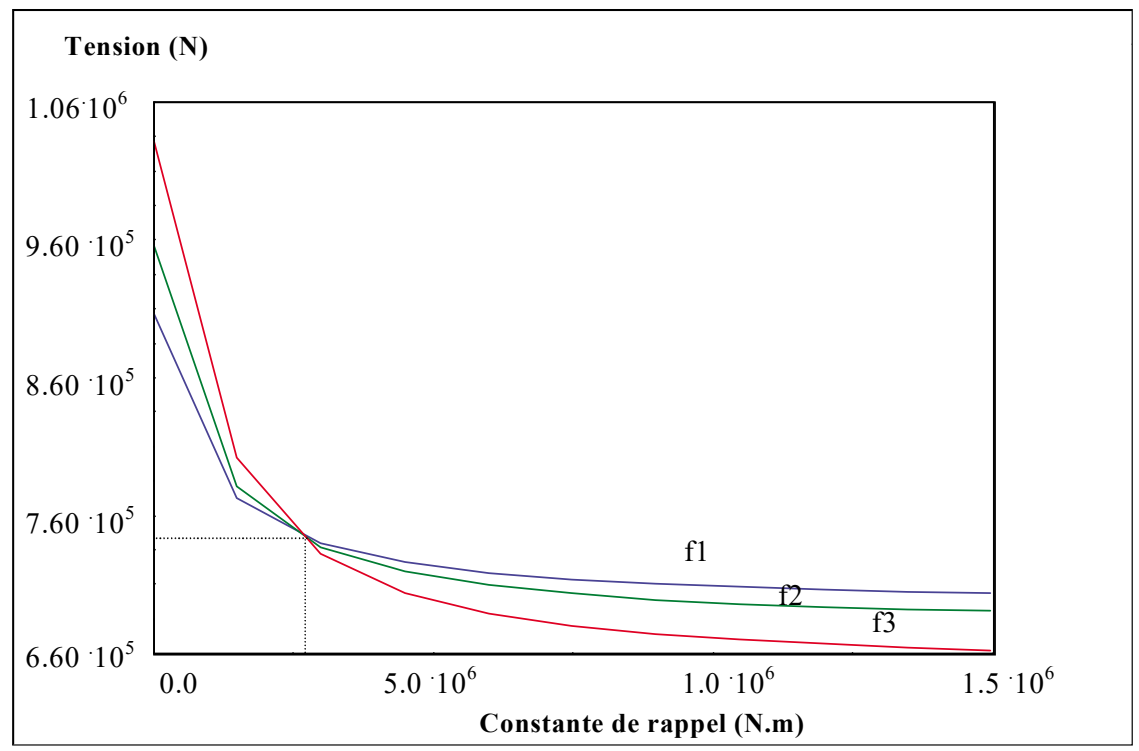

Figure 9. Résolution graphique du problème inverse - représentation des isovaleurs des trois premières fréquences

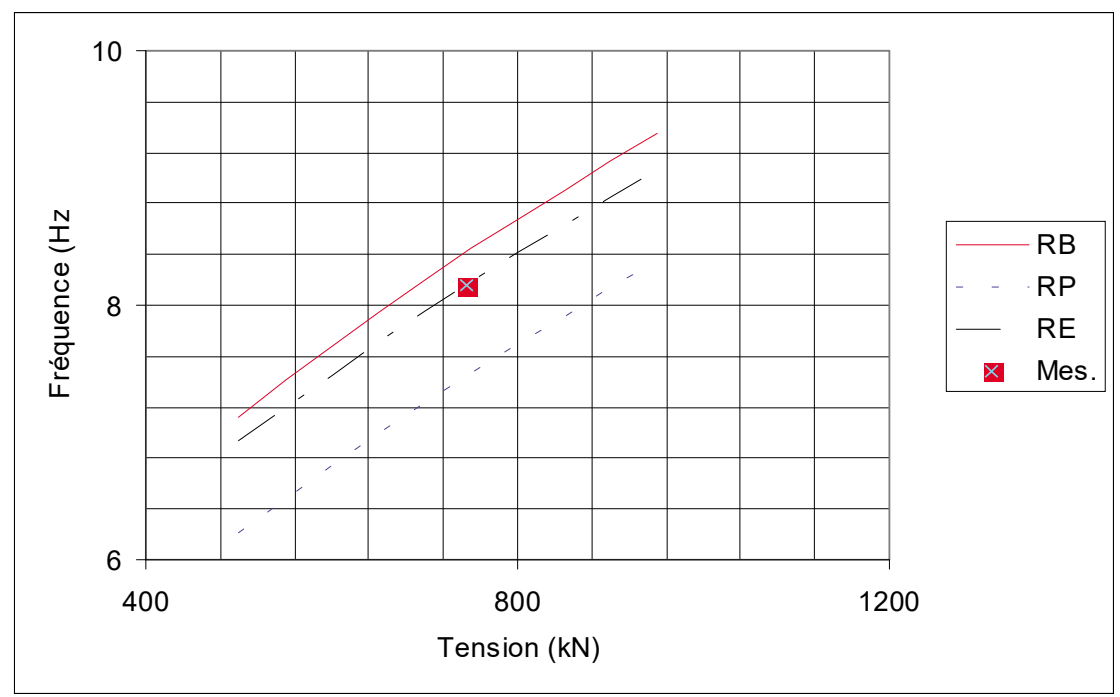

Figure 10. Relations entre la première fréquence de vibration et la tension pour les cas limites de conditions relatives au fonctionnement de la rotule : blocage parfait (RB) - articulation sans frottement, RE : rotule partiellement bloquée, Mes : point de fonctionnement

La figure 10 montre les relations théoriques entre la tension et la première fréquence propre pour les deux cas limites de fonctionnement du dispositif d'ancrage inférieur: parfaitement bloqué et parfaitement articulé. Le fonctionnement du dispositif d'ancrage partiellement bloqué y est également représenté avec l'état du système déterminé par le calcul de recalage. L'erreur sur la détermination de la tension en supposant l'ancrage parfaitement bloqué est égale à $8 \%$. Le recalage avec trois paramètres à déterminer, incluant la rigidité à la flexion $\mathrm{du}$ toron, permet de réduire de manière sensible l'écart entre les six premières 
fréquences mesurées et calculées. Les résultats obtenus à partir des 6 premières fréquences propres sont présentés dans le tableau 5. Les valeurs des paramètres corrigés du modèle sont rapportés dans le tableau 6 et comparées aux valeurs obtenues par recalage avec deux paramètres.

\begin{tabular}{|l|c|c|c|}
\hline & $\begin{array}{c}\mathrm{C} \\
{[\mathrm{N} . \mathrm{m}]}\end{array}$ & $\begin{array}{c}\text { EI } \\
{\left[\mathrm{kN} . \mathrm{m}^{2}\right]}\end{array}$ & $\begin{array}{c}\mathrm{T} \\
{[\mathrm{kN}]}\end{array}$ \\
\hline Recalage 2 paramètres & $2,5 \cdot 10^{6}$ & 239 & 746 \\
\hline Recalage 3 paramètres & $1,1 \cdot 10^{7}$ & 187,5 & 735 \\
\hline
\end{tabular}

Tableau 6. Fréquences de vibration mesurées et calculées

La valeur obtenue de la rigidité à la flexion du toron est plus faible que la valeur maximale estimée. En effet, au niveau de l'ancrage le toron est épanoui pour réaliser l'opération de culottage. Les fils ne sont probablement plus tous en contact. De plus, notre modèle suppose que l'encastrement du toron est parfait. En fait, la valeur du module de flexion obtenue après recalage intègre l'effet d'encastrement partiel. La valeur de la tension déterminée par la méthode du recalage de modèle à trois paramètres est égale à $735 \mathrm{kN}$, soit une valeur inférieure de $1,5 \%$ à celle obtenue après recalage avec deux paramètres.

\subsection{Hauban d'une passerelle}

La dernière étude de cas présentée concerne la détermination de la tension d'un hauban de passerelle qui a également été instrumenté et dont la longueur est proche de $30 \mathrm{~m}$. La figure 11 montre une photographie du dispositif d'ancrage bas qui correspond au dispositif réglable. Le dispositif d'ancrage de la partie supérieure est un culot d'ancrage à chape court et moins massif. Les caractéristiques du modèle représenté sur la figure 12 sont rapportées dans le tableau 7.

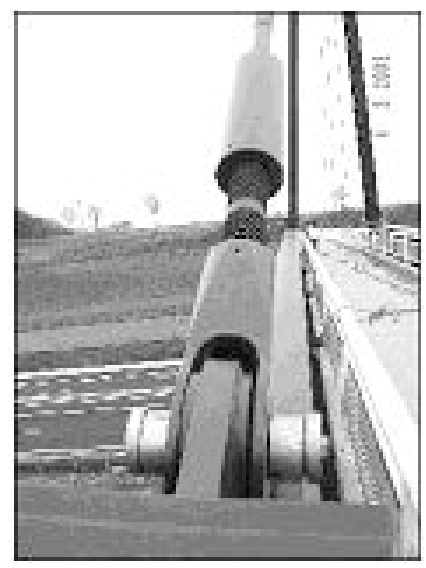

Figure 11. Dispositif d'ancrage bas réglable 
Comme pour le cas de la suspente courte traitée dans le paragraphe 3.2, les fréquences propres mesurées dans le plan de l'axe de la chape et dans le plan perpendiculaire sont identiques. Les résultats des mesures sont rapportés dans le tableau 8.

\begin{tabular}{|c|c|c|c|c|c|c|c|}
\hline \multicolumn{4}{|c|}{ Toron } & \multicolumn{4}{c|}{ Dispositifs d'ancrage } \\
\hline $\begin{array}{c}\text { Longueur } \\
\text { L }[\mathrm{m}]\end{array}$ & $\begin{array}{c}\text { Diamètre } \\
\mathrm{D}[\mathrm{mm}]\end{array}$ & $\begin{array}{c}\text { Masse } \\
\text { linéique } \\
\mathrm{M}[\mathrm{kg} / \mathrm{m}]\end{array}$ & $\begin{array}{c}\text { Rigidité à } \\
\text { la flexion } \\
\text { EI }\left[\mathrm{N} \cdot \mathrm{m}^{2}\right]\end{array}$ & $\begin{array}{c}\text { Moment } \\
\mathrm{d}^{\prime} \text { inertie } \\
\mathrm{J}_{1}\left[\mathrm{~kg} \cdot \mathrm{m}^{2}\right]\end{array}$ & $\begin{array}{c}\text { Longueur } \\
\mathrm{l}_{1}[\mathrm{~m}]\end{array}$ & $\begin{array}{c}\text { Moment } \\
\mathrm{d}^{\prime} \text { inertie } \\
\mathrm{J}_{2}\left[\mathrm{~kg} \cdot \mathrm{m}^{2}\right]\end{array}$ & $\begin{array}{c}\text { Longueur } \\
1_{2}[\mathrm{~m}]\end{array}$ \\
\hline 27,16 & 53 & 18,20 & 77465 & 5 & 0.38 & 53 & 1,11 \\
\hline
\end{tabular}

Tableau. Caractéristiques du hauban

\begin{tabular}{|c|c|c|c|c|c|c|}
\hline & $\mathrm{f} 1$ & $\mathrm{f} 2$ & $\mathrm{f} 3$ & $\mathrm{f} 4$ & $\mathrm{f} 5$ & $\mathrm{f6}$ \\
\hline $\begin{array}{c}\text { Mesure } \\
\text { (résolution) }\end{array}$ & 3,34 & $\begin{array}{c}6,60- \\
6,68 \\
(0,02)\end{array}$ & $\begin{array}{c}10,00 \\
(0,02)\end{array}$ & $\begin{array}{c}13,32 \\
(0,02)\end{array}$ & $\begin{array}{c}16,84 \\
(0,1)\end{array}$ & $\begin{array}{c}20,28- \\
20,44 \\
(0,1)\end{array}$ \\
\hline $\begin{array}{c}\text { Calcul après } \\
\text { recalage }\end{array}$ & 3,33 & 6,66 & 10,02 & 13,41 & 16,84 & 20,32 \\
\hline
\end{tabular}

Tableau 8. Fréquences mesurées et calculées après recalage du modèle (T,C,EI)

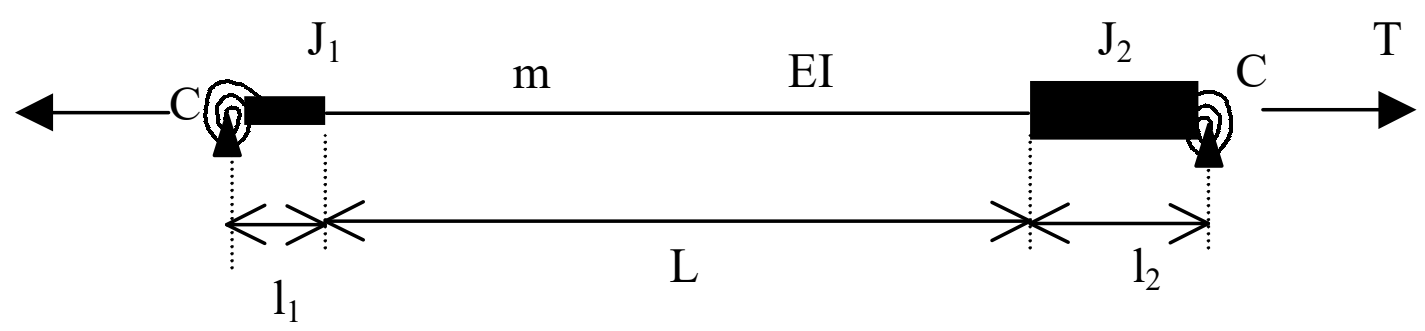

Figure 12. Modèle mécanique du hauban

La valeur de la tension calculée avec le modèle de la corde vibrante en supposant que les articulations des dispositifs d'ancrage fonctionnent parfaitement est égale à $667 \mathrm{kN}$. La valeur du paramètre de flexion est alors égale à $1,4 \cdot 10^{-4}$ et pour une poutre parfaitement articulée aux deux extrémités le modèle de la corde vibrante donne une excellente approximation de la relation entre la tension et la première fréquence propre. En supposant les dispositifs d'ancrage parfaitement bloqués, le modèle de la corde vibrante donne une tension égale à $599 \mathrm{kN}$, mais cette valeur est surestimée pour ces conditions de fonctionnement car l'effet de la rigidité à la 
flexion du toron parfaitement encastré n'est plus négligeable. Dans le modèle représenté sur la figure 12, on a supposé les constantes élastiques de rappel identiques pour les deux dispositifs d'ancrage car les systèmes d'articulation sont identiques. Après recalage du modèle avec les trois paramètres T, C et EI, la valeur de la tension obtenue est égale à $571 \mathrm{kN}$. La valeur de la rigidité à la flexion recalculée est pratiquement diminuée de moitié par rapport à la valeur maximale estimée. Les résultats du calcul de recalage sont rapportés dans le tableau 9.

\begin{tabular}{|c|c|c|c|}
\hline & $\begin{array}{c}\mathbf{C} \\
{[\mathbf{N} . \mathbf{m}]}\end{array}$ & $\begin{array}{c}\mathbf{E I} \\
{\left[\mathbf{k N} \cdot \mathbf{m}^{2}\right]}\end{array}$ & $\begin{array}{c}\mathbf{T} \\
{[\mathbf{k N}]}\end{array}$ \\
\hline $\begin{array}{c}\text { Recalage } \\
\text { 3 paramètres }\end{array}$ & $5 \cdot 10^{8}$ & 43,3 & 571 \\
\hline
\end{tabular}

Tableau 9. Recalage des paramètres du modèle

Les valeurs des fréquences propres calculées sont comparées aux valeurs expérimentales dans le tableau 8. Compte tenu de la résolution de la détermination expérimentale des fréquences, l'accord entre les valeurs mesurées et calculées est satisfaisant.

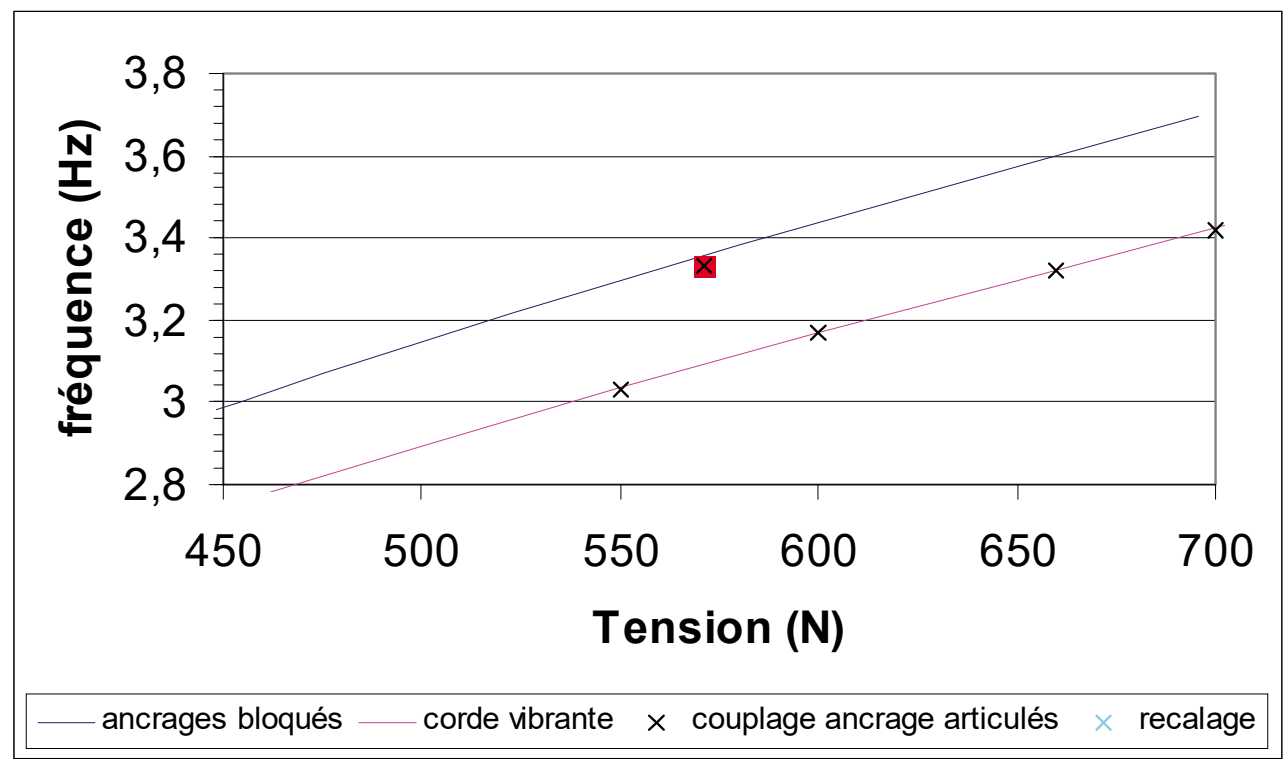

Figure 13. Relations entre la tension et la première fréquence pour les différents modèles

Les relations entre la fréquence et la première fréquence propre obtenues pour les différents modèles simples : poutre encastrée au niveau des dispositifs d'ancrage parfaitement bloqués et corde vibrante avec des dispositifs d'ancrage parfaitement articulés, sont représentées sur la figure 13. Le point de fonctionnement déterminé pour le modèle recalé y est également représenté. On constate que d'après les résultats du recalage du modèle, l'articulation est plus proche du fonctionnement bloqué pour l'excitation qui a été appliquée. 


\section{Conclusions}

Des exemples de cas de détermination de la tension dans des systèmes de précontrainte, de haubanage ou de suspension à partir des fréquences de vibration mesurées ont été présentés. Les longueurs des systèmes tendus considérés étaient trop courtes pour pouvoir utiliser le modèle de la corde vibrante pour déterminer la tension. Des modèles mécaniques pour représenter de manière simplifiée les conditions d'ancrage par des liaisons élastiques et la distribution d'inertie ont été proposés. L'application de la technique de recalage local de modèle par la méthode de sensibilité à partir des premières fréquences propres a montré des résultats encourageants pour étendre le domaine d'application de la méthode LPC $\mathrm{n}^{\circ} 35$ aux systèmes courts. Un nombre réduit de paramètres à corriger a été utilisé dans les exemples présentés pour tenir compte des conditions d'encastrement et du fonctionnement des dispositifs d'ancrage susceptibles d'être articulés.

La mesure de fonctions de réponse en fréquence avec par exemple la technique du marteau instrumenté, qui est adaptée aux mesures à l'extérieur du laboratoire, permettrait de compléter notre connaissance du fonctionnement dynamique des éléments étudiés. On pourrait ainsi vérifier la linéarité de la réponse à l'excitation appliquée et augmenter si nécessaire le nombre de paramètres à identifier pour raffiner le modèle mécanique utilisé. Pour le suivi de l'évolution du fonctionnement du système, l'intérêt de l'identification des paramètres d'un modèle mécanique est également à souligner. Compte tenu de la sensibilité des fréquences propres aux conditions d'ancrage qui constituent des sites privilégiés d'endommagement, le recalage de modèle permet de préciser l'origine mécanique des changements observés au niveau du comportement vibratoire.

\section{Bibliographie}

Kevorkian J., Cole J.D., Perturbation Methods in Applied Mathematics, Springer-Verlag, 1981.

Livingston T., Béliveau J.G., Huston D.R., "Estimation of axial load in prismatic menbers using flexural vibrations", Journal of Sound and Vibration, vol. 179, n ${ }^{\circ}$, 1995, p. 899-908.

Mars (de) Ph., Hardy D., « Mesure des efforts dans les structures à câbles », Ann. T.P., vol. 6, Belgique, 1985, p. 515-531.

Morse, Ingard, "Theoretical acoustic", Mc Graw Hill, 1968, p.187-191.

Robert J.L., Bruhat D., Gervais J.P., «Mesure de la tension des câbles par méthode vibratoire », Bulletin de Liaison des Ponts et Chaussées, n 173, mai-juin 1991, p. 109-114.

Robert J.L., « Mesure de la tension des câbles par vibration », Méthode d'essai LPC n 35, 1993.

SETRA, «Comportements dynamique des haubans », Recommandations de la Commission Interministérielle de Précontrainte, Haubans, Chapitre 8, 2001. 
The Akashi-Kaikyo bridge: Design and construction of the world's longest bridge, HonshuShikoku bridge authority, Japan, 1998.

Valette C., Cuesta C., « Mécanique de la corde vibrante », Traité des nouvelles technologies. Série mécanique, Hermès, 1993. 